\title{
Research on Reactive Power Compensation Optimization of 35kV Three Phase Unbalanced Distribution Network System
}

\section{Gongxing Chen}

School of Electrical \& Electronic Engineering, Tianhe College of Guangdong Polytechnical Normal University, Guangzhou, China

\section{Email address:}

1638839862@qq.om

\section{To cite this article:}

Gongxing Chen. Research on Reactive Power Compensation Optimization of 35kV Three Phase Unbalanced Distribution Network System. Science Journal of Circuits, Systems and Signal Processing. Vol. 7, No. 2, 2018, pp. 60-67. doi: 10.11648/j.cssp.20180702.13

Received: April 16, 2018; Accepted: May 2, 2018; Published: May 22, 2018

\begin{abstract}
In view of the actual situation of three-phase load unbalance in distribution power system, a three-phase load balancing compensation method for three-phase-and-three-wire systems in distribution network is proposed, and load model is established. Then I have deduced the mathematical model of three-phase load compensation in distribution network, and analyzed the energy loss of the two kinds of compensation method about the power supply and the dispersion compensation on the load spot. It is verified that the load end compensation can contribute more to the reduction of energy loss, under the premise of ensuring active load, possess more economical, this method has important significance for the solution in research on reactive power compensation optimization of three phase unbalanced distribution power network.
\end{abstract}

Keywords: Distribution Network, Three Phase Unbalanced, Load Compensation, Optimization

\section{Introduction}

The scale of electrical energy production is very large, and the transmission of electrical energy mainly depends on line transmission. Because of the impedance of the line, it often results in the loss of a lot of electrical energy during the transmission process, and at the same time, there is a voltage drop on the line. When the three-phase load is seriously unbalanced, the voltage at the end of the line will be lower than the national specific regulations.

In addition, with the rapid development of power electronics influx into the power system, the non-linear load in the distribution network has increased significantly. The situation of the unbalanced three-phase load of the distribution network is increasingly intensified, and the gap between the three-phase currents is significantly increased, this seriously reduces the quality of electrical energy.

The unbalance of three-phase load will result in the of reduction the utilization ratio of a power distribution network and the extreme unbalance of three-phase voltage and current. When the imbalance is very bad, it will endanger the safety operation of the entire power system and the power equipment. Reactive power compensation technology for power grid can not only improve the power factor of the power grid, but also improve the three-phase unbalanced case of the power grid. Therefore, along with the social progress, development of power grids and the improvement of users' requirements for power quality, it is extremely necessary to study the three-phase unbalanced reactive power compensation technology of power grids. According to the characteristics of the distribution network, reactive power optimization compensation is made for three-phase unbalanced load in distribution network. Whether it is from the perspective of power quality and power grid operation, or engineering needs, it is of great significance [1-3].

The reactive power optimization of distribution network is studied less under the consideration of three-phase unbalanced conditions. This paper considers the three-phase imbalance of the load in the distribution network and studies the optimal compensation method for the loss. The three-phase load is adjusted in order to balance the three-phase current. It is an important measure to reduce power consumption and save energy. It has good economic benefits.

\section{Method}

\subsection{Asymmetric Load Compensation and Derivation}

No matter what the form of asymmetry is, we must adopt the following three ways to achieve balance control in order to achieve the balance compensation of the system.

First, the current source with adjustable amplitude and phase can be injected into the circuit, and the current balance can be achieved through current synthesis. This method is usually achieved by connecting capacitors or inductors on the line. The balance compensation method has the lowest cost and can be widely applied in distribution network, but the 
effect of compensation must be optimized.

Second, a voltage source that can be adjusted in the line with an amplitude and a phase changes the amplitude and the phase of the load voltage through the vector of voltage to achieve the balance of the three-phase line current. Generally, different phase voltage sources are obtained by electromagnetic coupling. This method is mainly applied to the principle of electromagnetic coupling to obtain different phase and amplitude of series voltage sources. The required equipment has a large volume, a heavy weight, and a large number of windings. It is not easy to achieve accurate balance compensation, but the reliability of the operation is generally higher.

Third, the balance compensation is realized through series and parallel combination, for example, the balanced power compensation control is realized by using the independent three-phase voltage output of the unified power flow controller UPFC. This method is one of the most promising methods at present. It can not only realize the balance compensation of intelligent reactive power, but also carry out active power transmission between different phases of the same line, but the cost is higher. As a whole, reactive compensation is used to achieve the balance compensation control of asymmetric system, which can achieve better balance effect. But the workload of compensation calculation is large. It can help to change the amplitude and phase of line current with the offset of equivalent neutral point of reactive power compensation branch. compensated branch is connected in parallel with the line voltage or phase voltage, so that the synthetic vector of the load current and the compensation current generates the phase offset. Then, with the help of the amplitude adjustment of the compensation current, the final synthetic current basically meets the symmetry requirement.

However, the compensation calculation workload is large. It can use the offset of the equivalent neutral point of the reactive compensation branch to change the amplitude and phase of the line current, which is equivalent to connecting the compensation line to the line voltage or phase voltage. The synthetic vector of load current and compensation current generates a phase shift, and then adjusts the magnitude of the compensation current so that the synthetic current substantially satisfies the requirement of symmetry.

In the three-phase-and-three-wire system shown in Figure 1 , the triangle-connected loads $Z_{a b}, Z_{b c}$, and $Z_{c a}$ have been converted into star-connected three-phase loads ZLa, ZLb, and ZLc, respectively, and their amplitudes and phases are all different. In order to correct the unbalanced three-phase load of the three-phase- and-three-wire system, it is assumed that the reactance values of the star connected balance compensation are respectively $\mathrm{xqa}, \mathrm{xqb}$ and $\mathrm{xqc}$ (positive value for inductance and negative value for capacitance). The purpose of balance compensation is to make amplitudes of three-phase currents $i_{a}(t), i_{b}(t)$ and $i_{c}(t)$ of three-phase power output equal or to make the phase angles of three currents the same. From the point of power factor compensation, the power factor of the phase can be equal to 1 as long as the reactance value in parallel can completely eliminate the reactive power absorbed or emitted by the corresponding phase load.

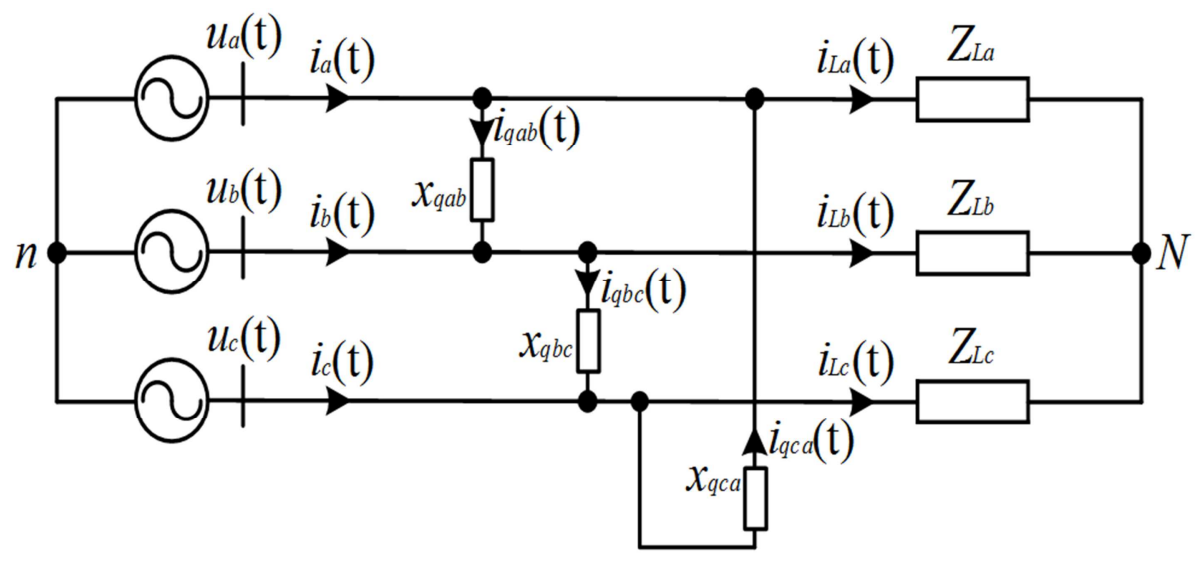

Figure 1. System compensation structure.

If this three-phase compensation method is used, there will be only active current in the system, but the current amplitude of system output may be asymmetrical, that is, The equivalent neutral point potential UN of the load side will shift. The principle of star balance compensation is to make compensation current vector generate the offset to some extent through the offset of the equivalent neutral point of the compensation structure. The angle between power supply voltage vector and synthesized current vector formed by load current and compensation current is as small as possible. At the same time, we should try to make the amplitude of the synthesized current as the same as possible. Under a certain asymmetric operation load, the vector diagram reflects the offset [4] of load neutral point potential shown in Figure 2. If the amplitude and phase of load current and compensation current are synthesized. And $\mathrm{C}$ phase power current is taken as an example, the amplitude and the phase are obtained in the dotted line in the lower left quarter shown in Figure 2. If the reactance amplitude of each compensation branch is changed, it is possible to satisfy the basic equilibrium condition through the synthesis effect of series and parallel connection of reactance devices. Even if we can't get the 
ideal balance compensation effect, we can carry out the control of the optimal balance compensation to make it near the condition of symmetrical operation. The influence of three phase compensation reactance on the balance compensation of three-phase-and-three-wire asymmetrical load is theoretically analyzed.

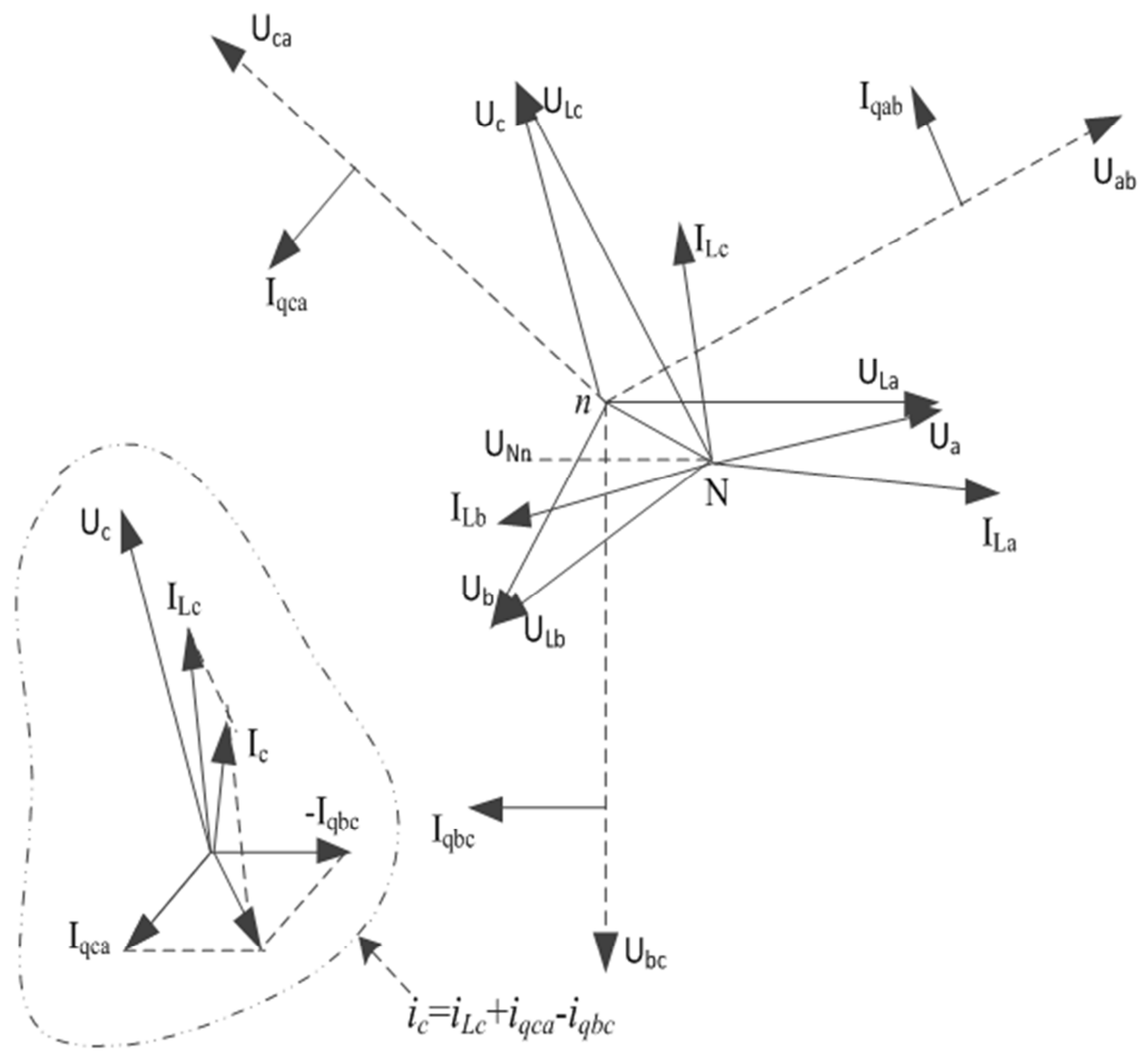

Figure 2. Vector diagram of balance compensation method.

Regardless of the line impedance, no matter what parallel compensation method is adopted, the active power generated by the power supply should remain unchanged before and after compensation.

After compensation at one or two locations in three compensation points of the unbalanced load, the current of the system is balanced to not consider whether the third group of load is symmetrical Compared with the three compensation points, at which the compensation device is invested, whether the line loss is smaller, that is, the active power output of the power supply will be smaller. There is greater economical efficiency.

Under the condition of the voltage symmetry of the three phase system, The asymmetrical current of the load is set for Iak, Ibk, Ick $(\mathrm{k}=1,2,3)$. In case of a compensation point which is put into three phase unbalanced compensation device among them, the three-phase current symmetry of the system is taken as the goal. The condition of the load that is not added to the compensator is unchanged. In practice, the admittance of the load is difficult to obtain, while the three phase line current and voltage can be collected in real time. Therefore, the symmetrical component method is used to represent the compensation susceptances by means of current and voltage. In the triangle compensation structure of Figure
1 , the equivalent impedance of three phase load is formulated as

$$
\begin{gathered}
\mathrm{ZLa}=\mathrm{rLa}+\mathrm{jxLa}=\mathrm{zLa} \angle \mathrm{La} \\
\mathrm{ZLb}=\mathrm{rLb}+\mathrm{jxLb}=\mathrm{zLb} \angle \mathrm{Lb} \\
\mathrm{ZLc}=\mathrm{rLc}+\mathrm{jxLc}=\mathrm{zLc} \angle \mathrm{Lc}
\end{gathered}
$$

The corresponding admittance is expressed as

$$
\begin{aligned}
& \mathrm{YLa}=1 / \mathrm{ZLa}=\mathrm{yLa} \angle-\mathrm{La} \\
& \mathrm{YLb}=1 / \mathrm{ZLb}=\mathrm{yLb} \angle-\mathrm{Lb} \\
& \mathrm{YLc}=1 / \mathrm{ZLc}=\mathrm{yLc} \angle-\mathrm{Lc}
\end{aligned}
$$

But the three-phase load is asymmetrical, and then $\mathrm{Za} \neq \mathrm{Zb} \neq \mathrm{Zc}, \mathrm{La} \neq \mathrm{Lb} \neq \mathrm{Lc}$.

For the compensation branch, we suppose that the compensation phase compensation is respectively $\mathrm{Yqab}=-\mathrm{jBqab}=1 / \mathrm{jxqab}, \quad \mathrm{Yqbc}=-\mathrm{jBqbc}=1 / \mathrm{jxqbc} \quad$ and Yqca $=-\mathrm{jBqca}=1 / \mathrm{jxqca}$.

According to Kirchhoff's current law and the current reference direction in the graph, the symmetrical component 
method is adopted. It is assumed that the phase voltage $[5,6$, 7] of the system is

$$
\left\{\begin{array}{l}
\dot{U}_{a}=\dot{U} \\
\dot{U}_{b}=a^{2} \dot{U} \\
\dot{U}_{c}=a \dot{U}
\end{array}\right.
$$

The symmetrical component of the line current is

$$
\left\{\begin{array}{l}
\dot{I}_{c 0}=0 \\
\dot{I}_{c 1}=j\left(B_{a b}+B_{b c}+B_{c a}\right) \dot{U} \sqrt{3} \\
\dot{I}_{c 2}=-j\left(a^{2} B_{a b}+B_{b c}+a B_{c a}\right) \dot{U} \sqrt{3}
\end{array}\right.
$$

The components of zero sequence and negative sequence flow over the compensation circuit as follows.

$$
\left\{\begin{array}{l}
\dot{I}_{c 0}=0 \\
\dot{I}_{c 1}=j\left(B_{a b}+B_{b c}+B_{c a}\right) \dot{U} \sqrt{3} \\
\dot{I}_{c 2}=-j\left(a^{2} B_{a b}+B_{b c}+a B_{c a}\right) \dot{U} \sqrt{3}
\end{array}\right.
$$

In the three-phase-and-three-wire system, the compensator should cancel the reactive part of the negative sequence component and the positive sequence component of the asymmetric current, so as to fully compensate for the three phase unbalance and reactive power.

$$
\left\{\begin{array}{l}
B_{a b}=-\frac{1}{3 \sqrt{3} U}\left[\operatorname{IM}\left(\dot{I}_{L 1}\right)+\operatorname{IM}\left(\dot{I}_{L 2}\right)-\sqrt{3} \operatorname{Re}\left(\dot{I}_{L 2}\right)\right] \\
B_{b c}=-\frac{1}{3 \sqrt{3} U}\left[\operatorname{IM}\left(\dot{I}_{L 1}\right)-2 \operatorname{IM}\left(\dot{I}_{L 2}\right)\right] \\
B_{c a}=-\frac{1}{3 \sqrt{3} U}\left[\operatorname{IM}\left(\dot{I}_{L 1}\right)+\operatorname{IM}\left(\dot{I}_{L 2}\right)+\sqrt{3} \operatorname{Re}\left(\dot{I}_{L 2}\right)\right]
\end{array}\right.
$$

The value of compensation admittance is obtained from the equations (6).

$$
\left\{\begin{array}{l}
B_{a b}=-\frac{1}{3 \sqrt{3} U}\left[\operatorname{IM}\left(\dot{I}_{L 1}\right)+\operatorname{IM}\left(\dot{I}_{L 2}\right)-\sqrt{3} \operatorname{Re}\left(\dot{I}_{L 2}\right)\right] \\
B_{b c}=-\frac{1}{3 \sqrt{3} U}\left[\operatorname{IM}\left(\dot{I}_{L 1}\right)-2 \operatorname{IM}\left(\dot{I}_{L 2}\right)\right] \\
B_{c a}=-\frac{1}{3 \sqrt{3} U}\left[\operatorname{IM}\left(\dot{I}_{L 1}\right)+\operatorname{IM}\left(\dot{I}_{L 2}\right)+\sqrt{3} \operatorname{Re}\left(\dot{I}_{L 2}\right)\right]
\end{array}\right.
$$

By changing to the phase coordinate system, there are

$$
\left\{\begin{array}{l}
B_{a b}=-\frac{1}{3 U}\left[\operatorname{IM}\left(\dot{I}_{L a}\right)+\operatorname{IM}\left(a \dot{I}_{L b}\right)-\operatorname{IM}\left(a^{2} \dot{I}_{L c}\right)\right] \\
B_{b c}=-\frac{1}{3 U}\left[-\operatorname{IM}\left(\dot{I}_{L a}\right)+\operatorname{IM}\left(a \dot{I}_{L b}\right)+\operatorname{IM}\left(a^{2} \dot{I}_{L c}\right)\right] \\
B_{c a}=-\frac{1}{3 U}\left[\operatorname{IM}\left(\dot{I}_{L a}\right)-\operatorname{IM}\left(a \dot{I}_{L b}\right)+\operatorname{IM}\left(a^{2} \dot{I}_{L c}\right)\right]
\end{array}\right.
$$

\subsection{Reactive Power Compensation Algorithm}

According to the characteristics of middle-low power distribution network, a compensation method that combines triangle connection compensation and star connection compensation is adopted. It not only can increase the power factor to 1, but also strive to achieve the minimum compensation capacity [8], under the premise of achieving the compensation effect. Before the power system is compensated, the voltage of each phase of a, b and c are $U_{a}$, $U_{b}, U_{c}$. The current of each phase are respectively $I_{a}, I_{b}, I_{c}$. And the power factor is respectively $\cos \theta_{a}, \cos \theta_{b}, \cos \theta_{c}$.

$$
\begin{array}{ll}
P_{a}^{L}=U_{a} I_{a} \cos \theta_{a}, & Q_{a}^{L}=U_{a} I_{a} \sin \theta_{a} \\
P_{b}^{L}=U_{b} I_{b} \cos \theta_{b}, & Q_{b}^{L}=U_{b} I_{b} \sin \theta_{b} \\
P_{c}^{L}=U_{c} I_{c} \cos \theta_{c}, & Q_{c}^{L}=U_{c} I_{c} \sin \theta_{c}
\end{array}
$$

In the case of three-phase three-wire system, the reactive power compensation equation for unbalanced loads is

$$
\begin{aligned}
& Q_{a}^{Y}=\frac{1}{\sqrt{3}} P_{b}^{L}-\frac{1}{\sqrt{3}} P_{c}^{L}+\frac{5}{6} Q_{a}^{L}-\frac{1}{6} Q_{b}^{L}-\frac{1}{6} Q_{c}^{L} \\
& Q_{b}^{Y}=\frac{1}{\sqrt{3}} P_{c}^{L}-\frac{1}{\sqrt{3}} P_{a}^{L}-\frac{1}{6} Q_{a}^{L}+\frac{5}{6} Q_{b}^{L}-\frac{1}{6} Q_{c}^{L} \\
& Q_{c}^{Y}=\frac{1}{\sqrt{3}} P_{a}^{L}-\frac{1}{\sqrt{3}} P_{b}^{L}-\frac{1}{6} Q_{a}^{L}-\frac{1}{6} Q_{b}^{L}+\frac{5}{6} Q_{c}^{L} \\
& Q_{a b}^{\Delta}=\frac{1}{3}\left[\frac{2}{\sqrt{3}} P_{a}^{L}-\frac{2}{\sqrt{3}} P_{b}^{L}+\frac{1}{6} Q_{a}^{L}+\frac{1}{6} Q_{b}^{L}+\frac{1}{6} Q_{c}^{L}\right] \\
& Q_{b c}^{\Delta}=\frac{1}{3}\left[\frac{2}{\sqrt{3}} P_{b}^{L}-\frac{2}{\sqrt{3}} P_{c}^{L}+\frac{1}{6} Q_{a}^{L}+\frac{1}{6} Q_{b}^{L}+\frac{1}{6} Q_{c}^{L}\right] \\
& Q_{c a}^{\Delta}=\frac{1}{3}\left[\frac{2}{\sqrt{3}} P_{c}^{L}-\frac{2}{\sqrt{3}} P_{a}^{L}+\frac{1}{6} Q_{a}^{L}+\frac{1}{6} Q_{b}^{L}+\frac{1}{6} Q_{c}^{L}\right]
\end{aligned}
$$

\section{Result}

The principle of three-phase unbalance compensation based on load compensation and the compensation method of load balance are derived, and the optimal compensation method is determined by analyzing and comparing the compensation line loss of power side and load side

\subsection{Power Supply Compensation}

In distribution line, because of its large scale and multiple points, the compensation capacity of every unbalanced point is limited. If every load is installed on the local compensation device, the system will be balanced and optimized [9, 10], but the requirement for the compensation device is to invest economically, safely and reliably. In Figure 3 , it is the equivalent compensation structure chart of the three way load and adopts the compensation mode of the power supply side.

Figure 3 shows the compensation structure at the power supply side. To compensate at the power supply side, the current is calculated at the power supply before the compensation. 


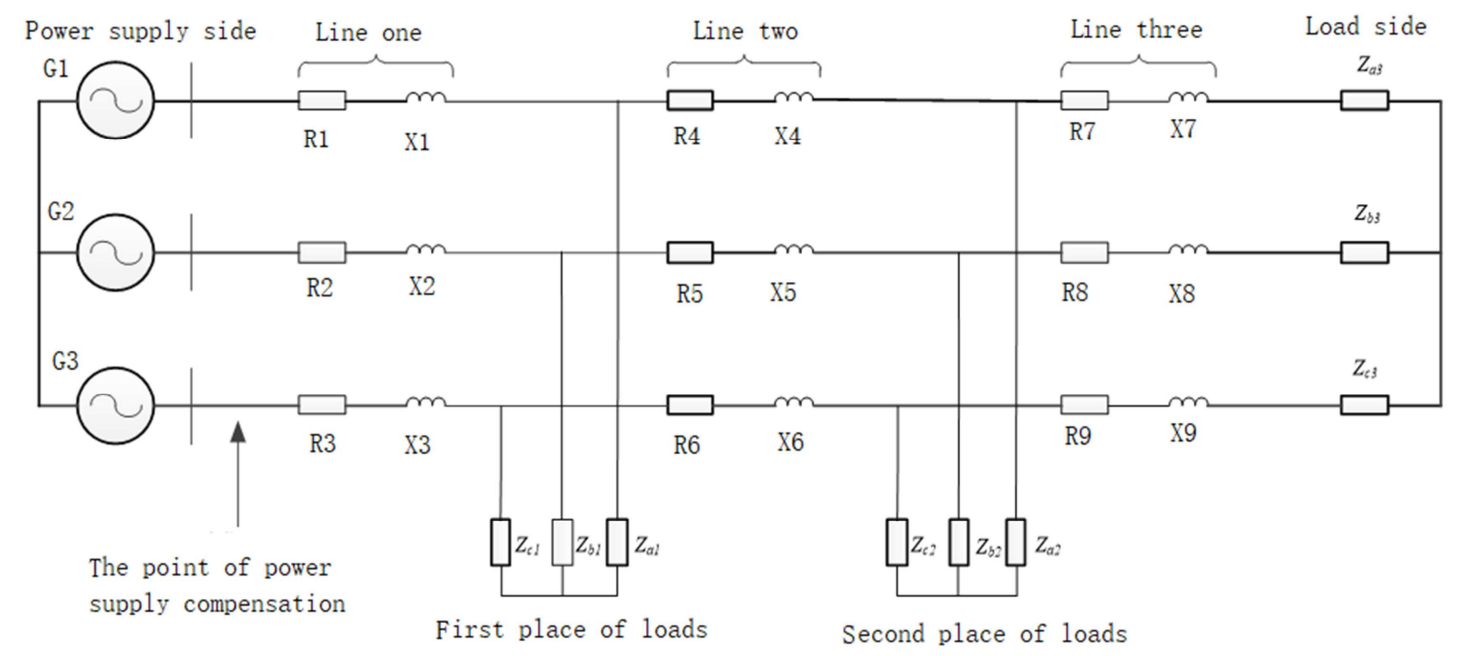

Figure 3. Structure diagram of power side compensation.

$$
\left.\begin{array}{l}
\dot{I}_{a}=\dot{I}_{L a 1}+\dot{I}_{L a 2}+\dot{I}_{L a 3} \\
\dot{I}_{b}=\dot{I}_{L b 1}+\dot{I}_{L b 2}+\dot{I}_{L b 3} \\
\dot{I}_{c}=\dot{I}_{L c 1}+\dot{I}_{L c 2}+\dot{I}_{L c 3}
\end{array}\right\}
$$

From equations (8), the admittance of compensation required by each branch is formulated as

$$
\begin{aligned}
& B_{a b}=-\frac{1}{3 U}\left[I M\left(\dot{\mathrm{I}}_{\mathrm{La} 1}+\dot{\mathrm{I}}_{\mathrm{La} 2}+\dot{\mathrm{I}}_{\mathrm{La} 3}\right)+\right. \\
& \left.I M\left(a \cdot\left(\dot{\mathrm{I}}_{\mathrm{Lb} 1}+\dot{\mathrm{I}}_{\mathrm{Lb} 2}+\dot{\mathrm{I}}_{\mathrm{Lb} 3}\right)\right)-I M\left(a^{2} \cdot\left(\dot{\mathrm{I}}_{\mathrm{Lc} 1}+\dot{\mathrm{I}}_{\mathrm{Lc} 2}+\dot{\mathrm{I}}_{\mathrm{Lc} 3}\right)\right)\right] \\
& B_{b c}=-\frac{1}{3 U}\left[-I M\left(\dot{\mathrm{I}}_{\mathrm{La} 1}+\dot{\mathrm{I}}_{\mathrm{La} 2}+\dot{\mathrm{I}}_{\mathrm{La} 3}\right)+\right. \\
& \left.I M\left(a \cdot\left(\dot{\mathrm{I}}_{\mathrm{Lb} 1}+\dot{\mathrm{I}}_{\mathrm{Lb} 2}+\dot{\mathrm{I}}_{\mathrm{Lb} 3}\right)\right)+I M\left(a^{2} \cdot\left(\dot{\mathrm{I}}_{\mathrm{Lc} 1}+\dot{\mathrm{I}}_{\mathrm{Lc} 2}+\dot{\mathrm{I}}_{\mathrm{Lc} 3}\right)\right)\right] \\
& B_{c a}=-\frac{1}{3 U}\left[I M\left(\dot{\mathrm{I}}_{\mathrm{La} 1}+\dot{\mathrm{I}}_{\mathrm{La} 2}+\dot{\mathrm{I}}_{\mathrm{La} 3}\right)-\right. \\
& \left.I M\left(a \cdot\left(\dot{\mathrm{I}}_{\mathrm{Lb} 1}+\dot{\mathrm{I}}_{\mathrm{Lb} 2}+\dot{\mathrm{I}}_{\mathrm{Lb} 3}\right)\right)+I M\left(a^{2} \cdot\left(\dot{\mathrm{I}}_{\mathrm{Lc1}}+\dot{\mathrm{I}}_{\mathrm{Lc} 2}+\dot{\mathrm{I}}_{\mathrm{Lc} 3}\right)\right)\right]
\end{aligned}
$$

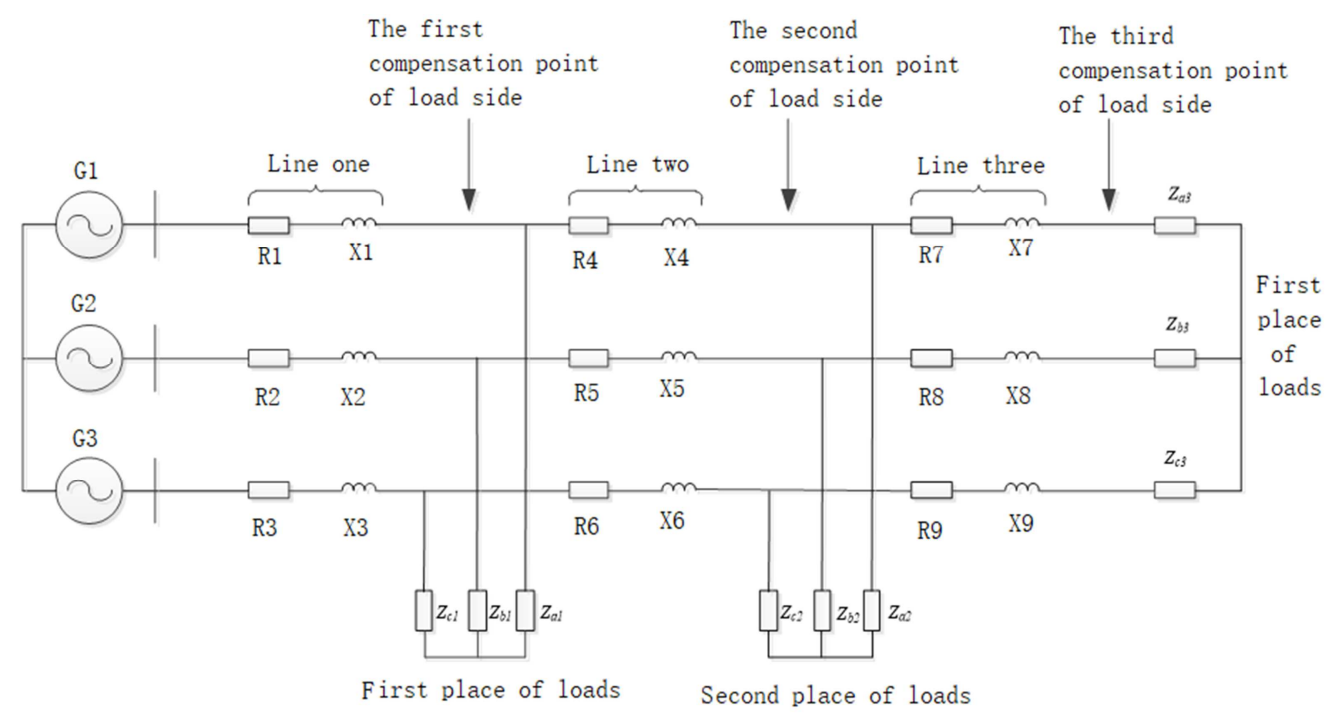

Figure 4. Structure diagram of each node load compensation.
After compensation, the current of the power supply side is computed as

$$
\left\{\begin{array}{l}
\dot{I}_{a}^{\prime}=\dot{I}_{a}+\dot{I}_{a 1} \\
\dot{I}_{b}^{\prime}=\dot{I}_{b}+\dot{I}_{b 1} \\
\dot{I}_{c}^{\prime}=\dot{I}_{c}+\dot{I}_{c 1}
\end{array}\right.
$$

\subsection{Load Side Compensation for Each Node}

In order to reliably ensure the compensation effect of each phase load and the power factor of the power supply [11], a reactive power compensation device needs to be installed at each node. Among them, It is the structural diagram of reactive power compensation at the load side of each node in Figure 4. 
Every admittance that needs compensation is formulated as

$$
\left\{\begin{array}{l}
B_{a b 1}=-\frac{1}{3 U}\left[\operatorname{IM}\left(\dot{I}_{L a 1}\right)+\operatorname{IM}\left(a \dot{I}_{L b 1}\right)-\operatorname{IM}\left(a^{2} \dot{I}_{L c 1}\right)\right] \\
B_{b c 1}=-\frac{1}{3 U}\left[-\operatorname{IM}\left(\dot{I}_{L a 1}\right)+\operatorname{IM}\left(a \dot{I}_{L b 1}\right)+\operatorname{IM}\left(a^{2} \dot{I}_{L c 1}\right)\right] \\
B_{c a 1}=-\frac{1}{3 U}\left[\operatorname{IM}\left(\dot{I}_{L a 1}\right)-\operatorname{IM}\left(a \dot{I}_{L b 1}\right)+\operatorname{IM}\left(a^{2} \dot{I}_{L c 1}\right)\right]
\end{array}\right.
$$

The current of one compensation branch is expressed as

$$
\left\{\begin{array}{l}
\dot{I}_{a_{-} 1}=\left(U_{a}-U_{b}\right) \cdot B_{a b 1} \cdot j-\left(U_{c}-U_{a}\right) \cdot B_{c a 1} \cdot i \\
\dot{I}_{b_{-} 1}=\left(U_{b}-U_{c}\right) \cdot B_{b c 1} \cdot j-\left(U_{a}-U_{b}\right) \cdot B_{a b 1} \cdot j \\
\dot{I}_{c_{-} 1}=\left(U_{c}-U_{a}\right) \cdot B_{c a 1} \cdot j-\left(U_{b}-U_{c}\right) \cdot B_{b c 1} \cdot j
\end{array}\right.
$$

One compensated symmetrical current is

$$
\left\{\begin{array}{l}
\dot{I}_{a_{-} 1}^{\prime}=\dot{I}_{a_{-} 1}+\dot{I}_{L a 1} \\
\dot{I}_{b_{-} 1}^{\prime}=\dot{I}_{b_{-} 1}+\dot{I}_{L b 1} \\
\dot{I}_{c_{-} 1}^{\prime}=\dot{I}_{c_{-} 1}+\dot{I}_{L c 1}
\end{array}\right.
$$

Similarly, the expression for two, three symmetrical current after compensation is

$$
\begin{gathered}
\left\{\begin{array}{l}
\dot{I}_{a-2}^{\prime}=\dot{I}_{a_{-} 2}+\dot{I}_{L a 2} \\
\dot{I}_{b_{-} 2}^{\prime}=\dot{I}_{b_{-} 2}+\dot{I}_{L b 2} \\
\dot{I}_{c_{-} 2}^{\prime}=\dot{I}_{c_{-} 2}+\dot{I}_{L c 2}
\end{array}\right. \\
\left\{\begin{array}{l}
\dot{I}_{a_{-} 3}^{\prime}=\dot{I}_{a_{-} 3}+\dot{I}_{L a 3} \\
\dot{I}_{b_{-} 3}^{\prime}=\dot{I}_{b_{-3} 3}+\dot{I}_{L b 3} \\
\dot{I}_{c_{-} 3}^{\prime}=\dot{I}_{c_{-} 3}+\dot{I}_{L c 3}
\end{array}\right.
\end{gathered}
$$

The current at the power side after compensation is

$$
\left\{\begin{array}{l}
\dot{I}_{a}^{\prime \prime}=\dot{I}_{a_{-} 1}+\dot{I}_{a_{-} 2}+\dot{I}_{a_{-} 3} \\
\dot{I}_{b}^{\prime \prime}=\dot{I}_{b_{-} 1}+\dot{I}_{b_{-} 2}+\dot{I}_{b_{-} 3} \\
\dot{I}_{c}^{\prime \prime}=\dot{I}_{c_{-} 1}+\dot{I}_{c_{-} 2}+\dot{I}_{c_{-} 3}
\end{array}\right.
$$

The power supply side compensation is compared with the current of the power supply after each node's load compensation as follows:

$$
\left\{\begin{array}{l}
\dot{I}_{a}^{\prime}=\dot{I}_{a}^{\prime \prime} \\
\dot{I}_{b}^{\prime}=\dot{I}_{b}^{\prime \prime} \\
\dot{I}_{c}^{\prime}=\dot{I}_{c}^{\prime \prime}
\end{array}\right.
$$

It can be seen that whether it is compensated at the power supply side or at the load of each node, it can be compensated. It can be seen that whether it is compensated at the power supply side or at the load of each node. The symmetrical current after compensation is always equal, then the power factor after the compensation is definitely the same. However, the difference is that the current of the two lines compensated are inconsistent, and the line losses are also inconsistent.

\section{Discussion}

\begin{tabular}{|c|c|c|c|c|c|}
\hline \multirow{2}{*}{ Group } & \multirow{2}{*}{ Phase } & \multicolumn{3}{|c|}{ Load compensation admittance /S } & \multirow{2}{*}{$\begin{array}{l}\text { Compensation admittance of } \\
\text { power side / } S\end{array}$} \\
\hline & & One & Two & Three & \\
\hline \multirow{3}{*}{ First group } & $\mathrm{AB}$ & -0.0021 & 0.0017 & -0.0026 & -0.0029 \\
\hline & $\mathrm{BC}$ & -0.0015 & 0.0032 & -0.0014 & 0.00027 \\
\hline & $\mathrm{CA}$ & 0.0042 & -0.00063 & 0.0032 & 0.0068 \\
\hline \multirow[b]{2}{*}{ Second group } & $\mathrm{AB}$ & 0.00024 & 0.00045 & 0.00011 & 0.0008 \\
\hline & $\mathrm{BC}$ & 0.0005 & 0.0023 & 0.00077 & 0.0036 \\
\hline \multirow{3}{*}{ Third group } & $\mathrm{AB}$ & 0.00063 & 0.0018 & 0.0017 & 0.0041 \\
\hline & $\mathrm{BC}$ & 0.0025 & 0.000574 & 0.0010 & 0.0041 \\
\hline & $\mathrm{CA}$ & 0.0016 & 0.0014 & 0.0014 & 0.0044 \\
\hline \multirow{3}{*}{ Fourth group } & $\mathrm{AB}$ & 0.0044 & -0.00046 & -0.00078 & 0.0032 \\
\hline & $\mathrm{BC}$ & 0.0023 & -0.00064 & -0.0010 & 0.0006 \\
\hline & $\mathrm{CA}$ & -0.0023 & 0.0014 & 0.00058 & -0.00035 \\
\hline
\end{tabular}

For convenience of calculations, the circuit impedance of each branch is assumed to be expressed as $\mathrm{Z}=\mathrm{R}+\mathrm{jX}$. Four groups of three-phase asymmetrical loads are respectively calculated by MATLAB software, and the two compensation admittance values above in Table 1 are obtained. The loss calculation equations are used to calculate the line loss of power side and each load node that are compensated as shown in Table 2, and to obtain the current of front and rear balance and the power factor after compensation and balance.

Table 1. Calculation of compensated admittance.

By analyzing and calculating load compensation admittance and power supply compensation admittance, load compensation admittance is beneficial to solve three-phase imbalance problem and compensation optimization. 


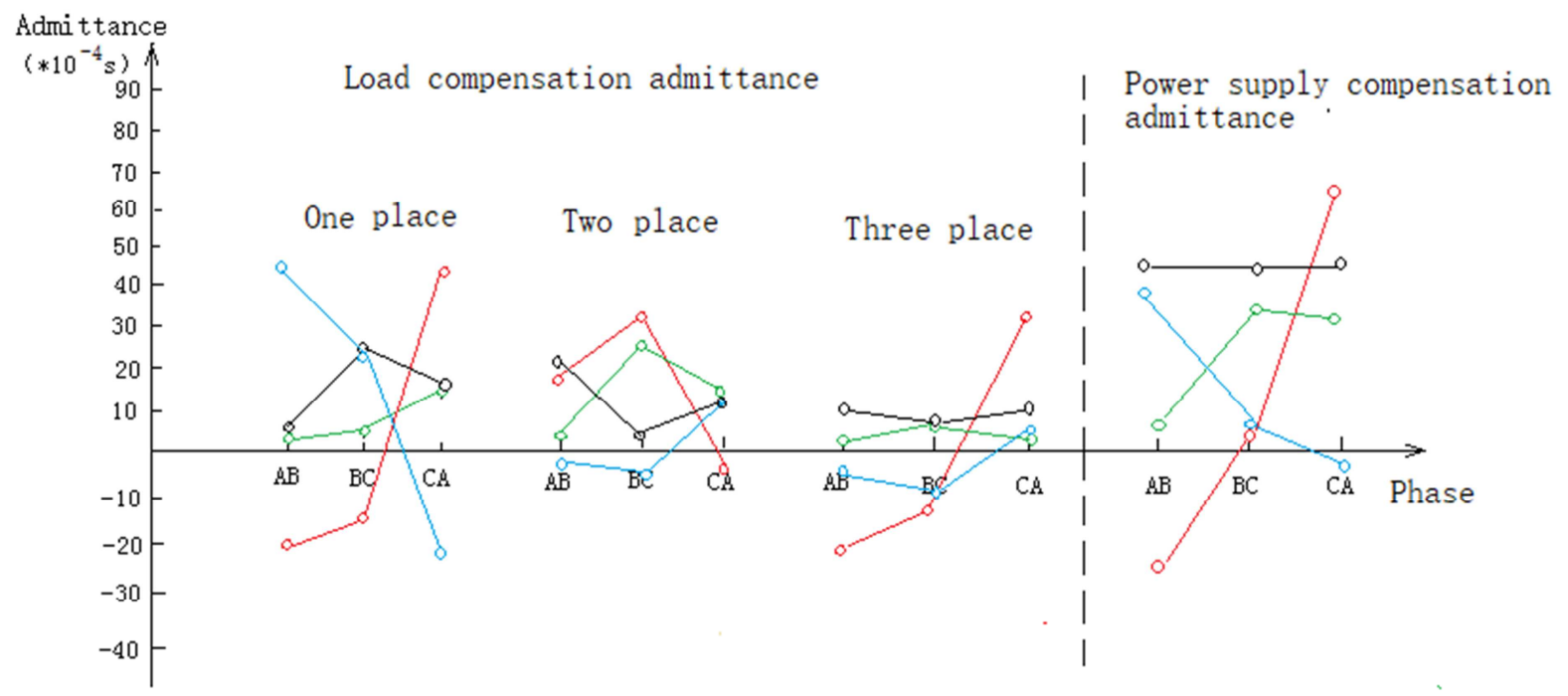

Figure 5. Admittance distribution chart of load compensation and power side compensation in distribution network.

Table 2. Comparison of line losses.

\begin{tabular}{|c|c|c|c|c|c|}
\hline Group & Phase & $\mathbf{Z}_{1} / \mathbf{\Omega}$ & $\mathbf{Z}_{2} / \mathbf{\Omega}$ & $\mathbf{Z}_{3} / \mathbf{\Omega}$ & Power supply current before compensation (Unit:A) \\
\hline \multirow{3}{*}{ First group } & A & $150 * \mathrm{j}$ & 100 & $200+10 * j$ & $120.2577 \angle-28.8329^{\circ}$ \\
\hline & B & $80-150 * j$ & $90+80 * j$ & $90-100 * j$ & $220.2855 \angle-109.7014^{\circ}$ \\
\hline & $\mathrm{C}$ & $90+80 * j$ & $70+200 * j$ & $80+60 * j$ & $267.2703 \angle 96.7751^{\circ}$ \\
\hline \multirow{3}{*}{ Second group } & A & $1+300 * j$ & $200+400 * j$ & $90+50 * \mathrm{j}$ & $65.2584 \angle-75.2851^{\circ}$ \\
\hline & B & $600+600 * \mathrm{j}$ & $50+200 * \mathrm{j}$ & $700+90 * j$ & $91.4704 \angle-166.1222^{\circ}$ \\
\hline & $\mathrm{C}$ & $70+300 * \mathrm{j}$ & $60+80 * j$ & $20+900 * \mathrm{j}$ & $111.6274 \angle 49.7586^{\circ}$ \\
\hline \multirow{3}{*}{ Third group } & A & $100+\mathrm{j} * 150$ & $70+\mathrm{j} * 80$ & $100+\mathrm{j} * 100$ & $176.5265 \angle-46.3708^{\circ}$ \\
\hline & B & $150+\mathrm{j} * 100$ & $170+j * 90$ & $140+\mathrm{j} * 130$ & $175.1651 \angle-164.8238^{\circ}$ \\
\hline & $\mathrm{C}$ & $20+\mathrm{j} * 180$ & $150+\mathrm{j} * 100$ & $130+\mathrm{j} * 120$ & $180.1036 \angle 74.8662^{\circ}$ \\
\hline \multirow{3}{*}{ Fourth group } & A & 40 & $300+90 * \mathrm{j}$ & $300+90 * j$ & $253.1355 \angle-9.6982^{\circ}$ \\
\hline & B & $80+70 * j$ & $80+80 * j$ & $80-70 * j$ & $204.5234 \angle-136.2131^{\circ}$ \\
\hline & $\mathrm{C}$ & $200+70 * j$ & $80-80 * j$ & $200-70 * j$ & $210.6857 \angle 119.0294^{\circ}$ \\
\hline
\end{tabular}

Table 2. Continue

\begin{tabular}{|c|c|c|c|c|c|c|}
\hline Group & Phase & $\begin{array}{l}\text { Power factor before } \\
\text { compensation }\end{array}$ & $\begin{array}{l}\text { Power side Current after } \\
\text { compensation (Unit:A) }\end{array}$ & $\begin{array}{l}\text { Power factor after } \\
\text { compensation }\end{array}$ & $\begin{array}{l}\text { Compensation line loss } \\
\text { of power side (Unit:W) }\end{array}$ & $\begin{array}{l}\text { Compensation line loss } \\
\text { of loader side(Unit:W) }\end{array}$ \\
\hline \multirow{3}{*}{$\begin{array}{l}\text { First } \\
\text { group }\end{array}$} & A & 0.8762 & $189.1947 \angle 0^{\circ}$ & 1 & \multirow{3}{*}{32576} & \multirow{3}{*}{18316} \\
\hline & $\mathrm{B}$ & 0.9839 & $189.1944 \angle-120.0608^{\circ}$ & 1 & & \\
\hline & $\mathrm{C}$ & 0.9190 & $189.1947 \angle 120.0609^{\circ}$ & 1 & & \\
\hline \multirow{3}{*}{$\begin{array}{l}\text { Second } \\
\text { group }\end{array}$} & A & 0.2547 & $39.2686 \angle-46.3708^{\circ}$ & 1 & \multirow{3}{*}{5407.1} & \multirow{3}{*}{892} \\
\hline & $\mathrm{B}$ & 0.6934 & $39.2686 \angle-46.3708^{\circ}$ & 1 & & \\
\hline & $\mathrm{C}$ & 0.3386 & $39.2686 \angle-46.3708^{\circ}$ & 1 & & \\
\hline \multirow{3}{*}{$\begin{array}{l}\text { Third } \\
\text { group }\end{array}$} & A & 0.6903 & $124.4149 \angle 0^{\circ}$ & 1 & \multirow{3}{*}{16782} & \multirow{3}{*}{8130.6} \\
\hline & B & 0.7096 & $124.4149 \angle-120.0608^{\circ}$ & 1 & & \\
\hline & $\mathrm{C}$ & 0.7057 & $124.4149 \angle 120.0609^{\circ}$ & 1 & & \\
\hline \multirow{3}{*}{$\begin{array}{l}\text { Fourth } \\
\text { group }\end{array}$} & A & 0.9857 & $218.8772 \angle 0^{\circ}$ & 1 & \multirow{3}{*}{34767} & \multirow{3}{*}{25444} \\
\hline & $\mathrm{B}$ & 0.9603 & $218.8770 \angle-120.0608^{\circ}$ & 1 & & \\
\hline & $\mathrm{C}$ & 0.9999 & $218.8772 \angle 120.0609^{\circ}$ & 1 & & \\
\hline
\end{tabular}

By comparing the current distribution of front and rear three-phase unbalanced reactive power compensation in distribution network, the current of the power supply side is stable after compensation and optimization. Thus, the power factor is increased to 1 , the line loss of load side compensation is far smaller than the line loss of power supply side compensation, and the safe operation of the distribution network is reliably guaranteed. 


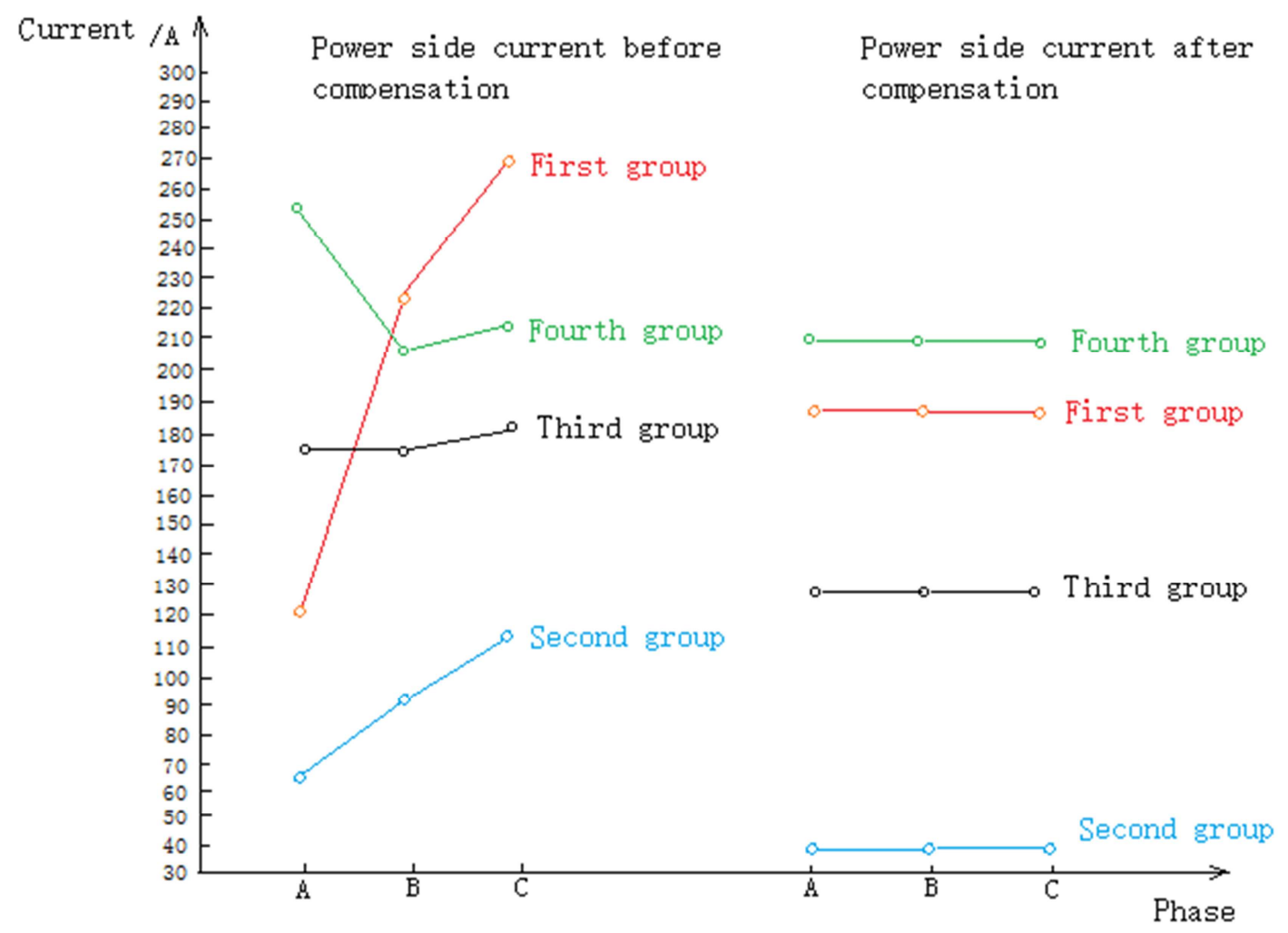

Figure 6. Currrent distribution chart of three phase unbalanced reactive power compensation in distribution network.

\section{Conclusion}

Through calculation and deduction, it can be seen that the load current of each node is equal to the current of each phase of the power side after the compensation. It is known by comparison and analysis of four groups of data in Table 1 and Table 2.

First, the two compensation methods can all achieve the effect of compensation current symmetry, help to solve the imbalance of the system, and compensate the power factor at the same time, and ensure the system's reactive demand.

Second, after analysis of line loss data in Table 2, it can be found that the actual loss reduction effect of local compensation at the load side of each node is better than power side compensation under the symmetry of two kinds of compensation systems. Under the premise of ensuring the active power of the load, the output of the active power of the power supply is relatively small, so it has better economic performance from the long-term investment effect. It is significant for solving the increasingly serious three-phase unbalanced optimization of distribution network in the future.

\section{References}

[1] Xuetao Fang. Research on reactive compensation technology for three-phase unbalanced load distribution network [D]. Southeast University, 2015, 6.

[2] Xiuqin Guo. Research on optimization scheme of energy saving and loss reduction in distribution network [J]. Power Capacitor and Reactive Compensation, 2014, 35(2):43-49.
[3] Xueqi Liao. Calculation Analysis and Loss Reduction Measures for Rural Network Line Loss [M]. Beijing: China Water Power Press, 2008.

[4] Hanxiang Cheng. Theory of reactive power compensation and its application [M]. Beijing: Machinery Industry Press, 2016.

[5] Qirong Jiang. Parallel Compensation of Power Systems-Structure, Principle, Control and Application [M]. Beijing: Mechanical Industry Press, 2004.

[6] Yinghong Hu. Analysis and compensation method of unbalanced load by balanced component method [J]. Proceedings of the CSEE, 2012, 34(12):98-104(in Chinese).

[7] Jianfei Dang. Effect of three-phase unbalanced operation on power grid and research on reactive power compensation method [J]. Electrical Engineering, 2012(8): 51-54.

[8] Min Xie, Yijiang Yin, Yuxin Du, Peijun Cheng, Mingbo Liu. Evaluation of available transfer capability of power system considering complex weather risk sources [J]. power automation equipment, 2018(4).

[9] Junwen Liang, Shunjiang Lin, Mingbo Liu. Distributed reactive power optimization control method in active distribution network [J]. power grid technology, 2017(7).

[10] Wentian Lu, Shunjiang Lin, Mingbo Liu. Active optimal allocation of liaison lines for $\mathrm{AC}$ and $\mathrm{DC}$ parallel transmission channels in long distance $[\mathrm{J}]$. Journal of South China University of Technology (NATURAL SCIENCE EDITION), 2017(7).

[11] Jun Zhao. Research on General Loss Reduction Method and Energy Saving Technology for $10 \mathrm{kV}$ Long Line System [D]. North China Electric Power University, 2013(6). 